\title{
Logros y Desafíos en la Formación de Competencias Transversales por áreas de Conocimiento en la Educación Superior del Instituto Politécnico Nacional (México)
}

\author{
Yasmín I. Jiménez, Juan J. Gutiérrez y Josefina Hernández \\ Instituto Politécnico Nacional, Escuela Superior de Cómputo, Departamento de Ingeniería en Sistemas \\ Computacionales, Av. Juan de Dios Bátiz s/n esq. Av. Miguel Othón de Mendizábal. Colonia Lindavista. Demarcación \\ Territorial: Gustavo A. Madero. C. P. 07738, México (e-mail yjimenezg@ipn.mx; jgutierrezg@ipn.mx \\ johernandezj@ipn.mx)
}

Recibido Jul. 19, 2018; Aceptado Oct. 1, 2018; Versión final Nov. 7, 2018, Publicado Jun. 2019

\begin{abstract}
Resumen
Esta investigación se centra en la valoración del desarrollo de competencias transversales de estudiantes de educación superior del Instituto Politécnico Nacional (México), que son componentes del perfil de egreso. El objetivo principal del estudio fue determinar, desde la percepción de los estudiantes, el desarrollo de competencias tales como: i) trabajo en equipo y actitud de liderazgo; ii) plan de vida y carrera; $y$ iii) conciencia y apertura al cambio. El estudio fue de tipo exploratorio y se aplicó un cuestionario a 1903 alumnos de tres áreas de conocimiento: Ingeniería y Ciencias Físico Matemáticas (ICFM), Ciencias Médico Biológicas (CMB) y Ciencias Sociales y Administrativas (CSA). Los resultados muestran que la competencia plan de vida y carrera de los estudiantes está ligada a una actuación autónoma. Se encuentra también que hay diferentes grados de desarrollo de la competencia trabajo en equipo y liderazgo, más desarrollada en estudiantes de CSA y menos en los de ICFM. Con relación a la conciencia y apertura al cambio el estudio muestra que en la conducta y valoración de diferentes actividades prevalecen roles y estereotipos.
\end{abstract}

\section{Achievements and Challenges in the Formation of Transversal Competences by Knowledge Areas in Higher Education of the National Polytechnic Institute (México)}

\begin{abstract}
This research focuses on the assessment of the development of transversal competences, part of the graduation profile, of higher education students of the National Polytechnic Institute (México). Its objective was to determine, from the perception of the students, the development of transversal competences such as: i) teamwork and leadership attitude; ii) Life and career plan; and iii) consciousness and openness to change, The study was of the exploratory-type and a questionnaire was applied to 1903 students from three areas of knowledge: Engineering and Physical and Mathematical Sciences (IPMS),Biological and Medical Sciences (BMS), and Social Sciences and Administration (SCA). The results show that the competition life and career plan of the students is linked to an autonomous performance It is also found that the existence of different degrees of competence development, teamwork and leadership- more developed in students of SCA and less in those of IPMS. With respect to consciousness and openness to change the study shows that conducts and valuation of different activities are still affected by social stereotypes.
\end{abstract}

Keywords: transversal competences; graduation student profile; higher education; educational research; team work; life plan; career plan 


\section{INTRODUCCIÓN}

El Informe Delors emitido por la Organización de las Naciones Unidad para la Educación, la Ciencia y la Cultura (UNESCO, 1996) estableció que la educación más allá de ser un derecho humano era un pasaporte para vivir con calidad y formuló como pilares de la misma cuatro saberes: conocer, hacer, ser y convivir. Esto implica para las instituciones educativas ir más allá del conocer y del hacer a través de los contenidos disciplinares habituales, para incluir aprendizajes ligados al desarrollo integral y armónico de los estudiantes (Bell, 2017; Tejeda 2016; Tawil y Cougoureux, 2013). El eje rector de los cuatro pilares es el aprendizaje a lo largo de la vida lo que posibilita a que cada estudiante desempeñe un rol activo para ensayar cosas nuevas, activar talentos latentes y lograr su realización (Tawil y Cougoureux, 2013). Estudios destacan que dicho Informe fue un marco filosófico para reorientar la agenda de los países de América Latina (AL) (Bell, 2017; Macías et al., 2017; Villarroel y Bruna, 2014), particularmente para las Instituciones de Educación Superior (IES) implicó la formación de profesionistas con el potencial de actuar con autonomía y con criterio como premisa para la elaboración de juicios propios ante sus decisiones más importantes -profesionales y personales- (Bell, 2017). Así, uno de los enfoques para la formación de profesionistas fue la Educación Basada en Competencias (EBC) del Proyecto Tuning AL (2004-2007). Dentro de la EBC la competencia hace referencia a la formación integral del ciudadano en las áreas cognoscitiva -saber conocer-, psicomotora saber hacer- y afectiva -saber ser y saber convivir- (Beneitone, et., 2007); o como un desempeño efectivo que activa de manera integrada conocimientos, habilidades, capacidades, destrezas, actitudes y valores, en diversos contextos (Tejeda, 2016; Villa y Poblete, 2011).

Los desempeños efectivos de los estudiantes se deben desarrollar por niveles de complejidad en dos tipos de competencias: específicas o profesionales y genéricas o transversales. Las competencias específicas son las que están íntimamente ligadas a las disciplinas o áreas de conocimiento que distinguen una profesión de otra; las competencias transversales son comunes a la mayoría de las profesiones (Beneitone et al. 2007), por lo que rebasan los límites de las disciplinas y deberán desarrollarse a través de todo el mapa curricular (Villa y Poblete, 2011).Las competencias transversales son transferibles -activadas- entre ámbitos profesionales y/o interpersonales (Villarroel y Bruna, 2014) por lo que tienen como características i) la multifuncionalidad para alcanzar diferentes objetivos, ii) la instrumentalidad para mejorar la calidad de vida, iii) la visión sistémica que permita la comprensión de las variables asociadas a la solución de problemas complejos, y d) una mayor complejidad mental porque involucran la activación de actitudes y valores (Tejeda, 2016; Villarroel y Bruna, 2014; Farías y Firinguetti, 2012). Las competencias transversales se clasifican en: instrumentales, interpersonales y sistémicas. En la tabla 1 se presentan un extracto de ellas.

Tabla 1: Tipos y dimensiones de las competencias transversales (Datos tomados de Muñoz, Medina, y Guillén, 2016; Villarroel y Bruna, 2014; Farías y Firinguetti, 2012)

\begin{tabular}{|l|l|}
\hline \multicolumn{1}{|c|}{ Tipo } & \multicolumn{1}{c|}{ Competencias consensadas } \\
\hline $\begin{array}{l}\text { Instrumentales: Capacidades cognitivas, } \\
\text { metodológicas y lingüísticas que permiten que los } \\
\text { egresados comprendan, construyan y actúen con } \\
\text { las particularidades de sus profesiones }\end{array}$ & $\begin{array}{l}\text { Capacidad de análisis y síntesis; Capacidad de planificación y } \\
\text { organización; Habilidad para el manejo de las Tecnologías de la } \\
\text { Información; Solución de problemas; Capacidad para tomar } \\
\text { decisiones }\end{array}$ \\
\hline $\begin{array}{l}\text { Interpersonales: Habilidades de orden social que } \\
\text { le permiten a los egresados integrarse en } \\
\text { diferentes colectivos y trabajar con efectividad. }\end{array}$ & $\begin{array}{l}\text { Habilidad para manejar conflictos; Capacidad crítica y } \\
\text { autocrítica; Habilidad para trabajar en equipos } \\
\text { interdisciplinarios; Ética profesional }\end{array}$ \\
\hline $\begin{array}{l}\text { Sistémicas: Cualidades individuales relacionadas } \\
\text { con la comprensión del funcionamiento global de } \\
\text { un conjunto o sistema. }\end{array}$ & $\begin{array}{l}\text { Aprendizaje autónomo; Capacidad de adaptación a nuevas } \\
\text { situaciones; Creatividad; Liderazgo; Autonomía; Diseño y } \\
\text { gestión de proyectos; Espíritu emprendedor }\end{array}$ \\
\hline
\end{tabular}

La declaración de la Educación de Calidad para Todos (UNESCO, 2007) -relacionada con el Informe Delorsretoma los principios educativos de pertinencia y significación para estudiantes de diferentes estratos sociales, culturas, capacidades e intereses. Esto exige transitar de una pedagogía homogénea hacia una flexible para enriquecer los procesos de enseñanza-aprendizaje y fortalecer el desarrollo personal y social de los alumnos; se destaca la importancia del desarrollo de las competencias transversales. Más recientemente, la UNESCO (2011) confirma la aspiración en formar a los profesionistas de manera tal que asuman un compromiso ciudadano y se conviertan en los protagonistas de sus vidas.

La formación de estudiantes para que se conviertan en protagonistas de sus vidas alude al concepto de igualdad, dentro del cual todos los seres humanos son libres para desarrollar sus capacidades personales y ser dueños de sus decisiones, sin ningún tipo de limitación impuesta por los estereotipos, los roles fijados en función del género o los prejuicios" (ONU Mujeres, 2011). De acuerdo con la Asociación Nacional de Instituciones de Educación Superior (ANUIES, 2015) en México la matrícula de las IES se encuentra sesgada por áreas de conocimiento: Educación; Salud; y Ciencias Sociales, Administrativas y Derecho están 
feminizadas; y masculinizadas: Ingeniería, Manufactura y Construcción; Servicios; Agronomía y Veterinaria; y Ciencias exactas y de la computación.

Las causas de elección de carrera descansan sobre la interpretación social y cultural de los roles y estereotipos -identidad- que marcan las conductas apropiadas o inapropiadas, tanto para hombres como para las mujeres, y fundamentan las creencias sobre las diferencias psicológicas y conductuales para cada uno de ellos (Gross, 2012; Sánchez, et al. 2011). Por otro lado, estudios que la división del trabajo ha sido configurada de manera dicotómica entre las esferas privada y pública, dando origen a lo que se conoce como trabajo reproductivo y de cuidado -introyectado por las mujeres-; y al trabajo productivo -introyectado por los hombres- influyendo entre otros en: planes de vida y carrera, tiempo dedicado al hogar, disponibilidad de tiempo libre (Zapata y Ayala, 2014; Buquet, 2011). Esto es aprendido desde la socialización primaria -al interior de la familia- aunque reforzado en la socialización secundaria -escuela- (Berger y Luckman, 2006). Los roles y estereotipos operan como estructuras mentales -ideas arraigadas- y propician que las personas tomen caminos preconcebidos por la sociedad. Bordieu (2008) acuñó el concepto del habitus para referirse al conjunto de esquemas de percepción, evaluación y acción del sujeto en la práctica cotidiana; enfatiza que estas estructuras se convierten en estructurantes muy eficientes de la conducta. Así, las mujeres y los hombres subjetivizan lo objetivo induciendo formas convencionales de pensar y actuar -elecciones de vida, carrera, comportamiento-. Adicionalmente, el Informe nacional sobre la violencia de género (2009) realizado por el Fondo de las Naciones Unidas para la Infancia (UNICEF) y la Secretaría de Educación Pública (SEP) concluye que los estereotipos tradicionales de género están más arraigados en niñas que en niños.

Diversas investigaciones enfatizan que la educación deberá asumir como principal tarea la transformación y el cambio de actitudes, valores y prácticas del estudiantado de tal forma que les permitan reformular su vida cotidiana; y coadyuvar a la construcción de alternativas de transformación de mitos, creencias y relaciones de poder, entre otros (Zapata y Ayala, 2014; Barffusón et al., 2010). Por otro lado, Le Pelley et al. (2010) y el INMUJERES (2008) manifiestan que para cambiar las construcciones sociales identitarias se debe actuar en varios niveles: la consciencia individual; las prácticas y convivencia familiar; el trabajo y en las instituciones públicas. Como se puede deducir, desde las IES se puede coadyuvar a los estudiantes a fortalecer sus competencias transversales -relacionadas con aspectos intra e interpersonales-. Macías et al. (2017) y Rojo y Navarro (2016) enfatizan la importancia de la adquisición de competencias transversales a través de los programas de tutorías. La tutoría de ninguna manera sustituye la docencia en el aula "sino que la enriquece y completa, siendo el reforzador necesario del apoyo integral a los alumnos... constituyéndose en la forma más adecuada de atención individualizada del estudiante en su proceso formativo" (Macías, et al. 2017:10). Asimismo, otras investigaciones se han abocado a determinar cuáles son las competencias transversales más importantes para el desarrollo personal y social (Vega, et al., 2017; Tejeda, 2016; Farías y Firinguetti, 2012) o más desatendidas por las IES (Bolívar, 2011) dentro de las cuales se encuentran: capacidad para tomar decisiones, trabajo colaborativo, gestión del tiempo, aprendizaje autónomo, capacidad emprendimiento, dominio personal, aprender a aprender, actuar con autonomía e iniciativa personal, capacidad de adaptación a nuevas situaciones, entre otras.

Esta investigación se centra en el desarrollo de las competencias transversales de los estudiantes del Instituto Politécnico Nacional (IPN) donde se promueve una formación integral y de alta calidad con base en el desarrollo del saber, del hacer, del ser y del estar contextualizados; desde el 2004 el perfil de egreso se reformuló para manifestar que: "Contarán con una sólida formación integral, con conocimientos generales científicos y tecnológicos, por lo que serán capaces de desempeñarse en distintos ámbitos combinando adecuadamente teoría y práctica en su campo profesional. Habrán adquirido las habilidades necesarias para desarrollar un plan de vida y carrera..., trabajar en equipo mostrando liderazgo. La formación del egresado estará sustentada en valores éticos y responsabilidad que los harán conscientes y abiertos al cambio, capaces de actualizarse permanentemente y de responder a las necesidades de la sociedad" (p.111). La oferta educativa del IPN para educación superior (ES) incluye 81 programas académicos divididos en tres ramas del conocimiento: Ingeniería y Ciencias Físico Matemáticas (ICFM) (65.00\%), Ciencias Médico Biológicas (CMB) $(24.00 \%)$ y Ciencias Sociales y Administrativas (CSA) (11.00\%) (IPN, 2015). Lo que podría indicar que en el estudiantado del IPN persiste la identidad que podría demeritar su capacidad de agencia para ampliar su visión a lo largo de su vida personal y profesional. La matrícula inscrita en modalidad presencial para los últimos años se muestra en la tabla 2.

Como se observa, existe una escasa variación por área de conocimiento en los tres últimos años. Considerando la importancia otorgada al desarrollo de competencias transversales para la potenciación del saber ser y el saber estar se estableció como objetivo de la investigación determinar el desarrollo de: trabajo en equipo y actitud de liderazgo; plan de vida y carrera; y conciencia y apertura al cambio que tienen los estudiantes de ES del IPN, con este fin se realizó una investigación exploratoria y se aplicó un cuestionario a 1903 alumnos de las tres áreas de conocimiento que se imparten en la institución. 
Tabla 2: Matrícula del Instituto Politécnico Nacional (Datos obtenidos de: https://goo.gl/XyBbPa)

\begin{tabular}{|l|r|r|r|r|r|r|}
\hline \multirow{2}{*}{ Ciclo escolar } & \multicolumn{2}{|c|}{$\begin{array}{c}\text { Ingeniería y Ciencias Físico } \\
\text { Matemáticas (ICFM) }\end{array}$} & \multicolumn{2}{c|}{$\begin{array}{c}\text { Ciencias Médico } \\
\text { Biológicas (CMB) }\end{array}$} & \multicolumn{2}{c|}{$\begin{array}{c}\text { Ciencias sociales y } \\
\text { administrativas (CSA) }\end{array}$} \\
\hline & Hombres & Mujeres & Hombres & Mujeres & \multicolumn{1}{c|}{ Hombres } & \multicolumn{1}{c|}{ Mujeres } \\
\hline $2014-2015$ & 70.37 & 29.63 & 38.88 & 61.12 & 42.89 & 57.09 \\
\hline $2015-2016$ & 73.01 & 26.99 & 39.24 & 60.76 & 44.46 & 55.54 \\
\hline $2016-2017$ & 72.44 & 27.56 & 39.60 & 60.40 & 45.09 & 54.91 \\
\hline
\end{tabular}

\section{MATERIALES Y MÉTODOS}

La investigación fue de carácter exploratorio pues se buscó el registro y el análisis e interpretación sobre las realidades del estudiantado para presentar una interpretación correcta de las mismas (Tamayo, 2003); se tomó en consideración las acciones de los estudiantes - plano conductual-y las opiniones o las creencias de su contexto escolar y familiar -plano actitudinal y afectivo- sobre: trabajo en equipo y liderazgo; plan de vida y carrera; y conciencia y apertura al cambio; las cuales forman parte de las competencias transversales de egreso de los estudiantes de educación superior del IPN.

Se eligió el cuestionario como instrumento de recolección de información y su diseño se estructuró en dos partes, en la primera se recabó información socio-demográfica de los estudiantes y en la segunda información específica de las categorías de análisis. En la tabla 3 se describe la estructura de la segunda parte del cuestionario de investigación.

Tabla 3: Diseño de la segunda parte del cuestionario

\begin{tabular}{|c|c|c|c|}
\hline Categoría de análisis & Información recogida & Tipo de reactivos & $\begin{array}{l}\text { Número de } \\
\text { reactivos }\end{array}$ \\
\hline \multirow{2}{*}{$\begin{array}{l}\text { a. Trabajo en equipo y } \\
\text { liderazgo. Competencia } \\
\text { interpersonal que le } \\
\text { permite a los egresados } \\
\text { integrarse efectivamente } \\
\text { en diferentes colectivos }\end{array}$} & $\begin{array}{l}\text { Plano conductual (la realidad, lo } \\
\text { que hacen en sus trabajos } \\
\text { académicos) }\end{array}$ & $\begin{array}{l}\text { Opción múltiple (Hombres - } \\
\text { Mujeres - Indistinto) }\end{array}$ & \multirow[b]{2}{*}{15} \\
\hline & $\begin{array}{l}\text { Plano actitudinal y afectivo } \\
\text { (opinión y creencias sobre el } \\
\text { desarrollo de sus proyectos } \\
\text { académicos) }\end{array}$ & $\begin{array}{l}\text { Opción múltiple (Hombres - } \\
\text { Mujeres - Indistinto) }\end{array}$ & \\
\hline \multirow{2}{*}{$\begin{array}{l}\text { c. Plan de vida y carrera. } \\
\text { Competencia instrumental } \\
\text { que les permite autonomía } \\
\text { para tomar decisiones } \\
\text { propias }\end{array}$} & $\begin{array}{l}\text { Visión a cinco años de egreso de } \\
\text { la carrera correspondiente }\end{array}$ & $\begin{array}{c}\text { Likert } \\
\text { Orden de preferencias a cinco } \\
\text { años }\end{array}$ & \multirow[b]{2}{*}{21} \\
\hline & $\begin{array}{l}\text { Plano actitudinal y afectivo } \\
\text { (opinión y creencias sobre su } \\
\text { proyecto de vida, tanto personal y } \\
\text { profesional) }\end{array}$ & $\begin{array}{l}\text { Likert } \\
\text { Totalmente de acuerdo - } \\
\text { totalmente en desacuerdo }\end{array}$ & \\
\hline \multirow{2}{*}{$\begin{array}{l}\text { d. Conciencia y apertura al } \\
\text { cambio. } \\
\text { Competencia sistémica } \\
\text { que les permite adaptarse } \\
\text { a nuevas situaciones o } \\
\text { contextos modificados }\end{array}$} & $\begin{array}{l}\text { Plano conductual (la realidad, lo } \\
\text { que hacen y lo que viven en sus } \\
\text { casas) }\end{array}$ & $\begin{array}{c}\text { Opción múltiple } \\
\text { (Hombres - Mujeres - Indistinto) }\end{array}$ & \multirow{2}{*}{17} \\
\hline & $\begin{array}{l}\text { Plano actitudinal y afectivo } \\
\text { (opinión y creencias sobre } \\
\text { cambios en el entorno) }\end{array}$ & $\begin{array}{l}\text { Likert } \\
\text { Totalmente de acuerdo - } \\
\text { totalmente en desacuerdo }\end{array}$ & \\
\hline
\end{tabular}

El diseño fue no experimental y la recolección de datos se realizó de septiembre a diciembre del 2017. La calidad del diseño siguió los criterios de precisión con el registro fidedigno de las opiniones de los expertos que validaron el cuestionario; y la confiabilidad con el registro riguroso de los pasos seguidos en la investigación desde su concepción hasta las técnicas empleadas (Babie, 2006).

El proceso de validación se realizó con la opinión de tres psicólogos. En un primer acercamiento se les dio una plática del contexto de la investigación explicándoles el objetivo y se les entregó impreso el marco teórico que sustenta la investigación y el cuestionario para su posterior análisis. Los criterios establecidos para la revisión y análisis del instrumento diseñado fueron tres: la utilidad $(U)$ del reactivo para medir competencias transversales (Trabajo en equipo y liderazgo, Plan de vida y carrera, y Conciencia y apertura al cambio valorada desde la identidad de género de los estudiantes; la representatividad $(R)$ de las preguntas para indicar la existencia de una correspondencia entre la pregunta y las definiciones operativas de la investigación 
de dichos constructos, y la factibilidad $(F)$ que se traducía en la probabilidad de que la pregunta fuera respondida apropiadamente por los estudiantes. Para cada uno de estos criterios se estableció una escala del 1 al 3 donde: 1 . El reactivo no es útil, representativo y/o factible; 2 . El reactivo presenta una utilidad, representatividad y/o factibilidad media o regular; y 3 . El reactivo es útil, representativo y/o factible. La retroalimentación obtenida de los expertos permitió validar que el instrumento desarrollado es útil, representativo, con validez interna y factible de ser comprendido por los estudiantes a los que fue dirigido.

Por ser un estudio exploratorio el método de muestreo fue no probabilístico y la técnica elegida correspondió a muestreo por cuotas estableciendo que la participación de los estudiantes fuera ser voluntaria y abarcar las tres áreas de conocimiento que se ofertan en el instituto. Los datos se ajustaron a una distribución normal, para determinar sus medias y varianzas; se utilizó una prueba de Anderson Darling y Ryan-Joiner de la que se obtuvieron los valores R-J entre 0.831 y 0.901 y un valor $p$ superior a 0.05 para la distribución de los alumnos por semestre, por edad y promedio. Para el diseño de las preguntas se buscó mantener una relación de equilibro entre las respuestas para satisfacer las condición de equidistancia entre las opciones y realizar un análisis de intervalos. En los casos que los datos presentaran una posible relación entre ellos se utilizaron las pruebas de chi-cuadrada y la V-Cramer para obtener un cuantificador de la relación.

\section{ANÁLISIS}

Tomando en cuenta la naturaleza exploratoria de la investigación los resultados presentados corresponden al total de la muestra analizada. El análisis se presenta en este aparatado dividido en: trabajo en equipo y liderazgo; plan de vida y carrera; y conciencia y apertura al cambio.

El cuestionario fue contestado por un total de 1903 alumnos -946 mujeres y 957 hombres- de los cuales 1138 fueron de ICFM; 238 de CMB y 527 de CSA. La taza de mujeres por cada hombre es: 0.54, 2.01 y 2.82 respectivamente para cada una de las áreas. La diferencia entre ellos corresponde a que el número de mujeres es menor en las áreas ICFM y mayor en CSA, como se describe en la tabla 2. Las edades de los alumnos se distribuyen con una media de 20.64 y una desviación de 1.41 años para las mujeres y para los hombres la media es 20.32 con desviación de 1.45. El rango de edades es entre 17 y 25 años. Un $19.00 \%$ de los alumnos trabaja, $7.00 \%$ de ellos son mujeres y $12.00 \%$ hombres. El $30.43 \%$ de los alumnos están en tercer semestre, $19.92 \%$ y 18.23 en quinto y primero los demás alumnos cursaban el resto de los semestres con porcentajes entre $1.26 \%$ y $6.94 \%$, esto debido a que el estudio se llevó a cabo en un semestre impar. El promedio académico de los alumnos en la muestra es de 7.97 con una varianza de 0.90 en general; en el caso de los hombres el promedio es 7.79 con varianza de 0.90 y para las mujeres 8.16 con varianza 0.85 .

\section{Trabajo en equipo y liderazgo}

Para explorar el plano conductual del trabajo en equipo y liderazgo, se les preguntó por cuál tipo de actividades prefieren realizar cuando trabajan en equipo: $39.67 \%$ prefieren las intelectuales; $31.27 \%$ de liderazgo; $17.87 \%$ organizativas; y $11.19 \%$ operativas. Al desagregar por $\mathrm{M} \mathrm{y} \mathrm{H}$ no hay cambio en el orden de preferencia de actividades, pero si en los porcentajes. Las M prefieren las intelectuales y de liderazgo con $33.83 \%$ para ambas mientras que los $\mathrm{H}$ prefieren intelectuales con $45.45 \%$ y de liderazgo $28.74 \%$. Las $\mathrm{M}$ tienen un $22.69 \%$ de preferencia por las de organización y $9.73 \%$ de operativas mientras que los $\mathrm{H}$ son $13.17 \%$ y $12.64 \%$. Esto es, las $\mathrm{M}$ no prefieren las actividades operativas y los $\mathrm{H}$ prefieren las intelectuales. Cuando se agrupan estas respuestas por área de conocimiento se observa que las áreas ICFM y CMB tienen un comportamiento similar y es diferente para CSA como se muestra en la Tabla 4.

Tabla 4: Preferencia por actividades para trabajar en equipo agrupado por área de conocimiento

\begin{tabular}{|l|c|c|c|}
\hline \multicolumn{1}{|c|}{ Actividad } & ICFM & CMB & CSA \\
\hline Intelectuales (Análisis, Diseño, Investigación) & $45.08 \%$ & $43.70 \%$ & $26.19 \%$ \\
\hline $\begin{array}{l}\text { De liderazgo (División del trabajo, comunicación general, resolución de } \\
\text { conflictos) }\end{array}$ & $29.00 \%$ & $33.61 \%$ & $35.10 \%$ \\
\hline De organización (Planificación de actividades y entregables) & $13.09 \%$ & $16.39 \%$ & $28.84 \%$ \\
\hline Operativas (Elaboración de informes, trabajo de campo) & $12.83 \%$ & $6.30 \%$ & $9.87 \%$ \\
\hline
\end{tabular}

Al calcular el valor de la $V$-Cramer se obtiene $V_{c}=0.232$ lo indica que hay evidencia de un efecto pequeño entre las variables; se sabe que esta diferencia no está relacionada el hecho de que haya más mujeres inscritas en CSA, ya que aquí hay un cambio de orden de preferencia (CSA prefiere liderazgo y CMB y ICFM intelectuales) que no aparece al agrupar por $\mathrm{H}$ y $\mathrm{M}$. 
Para explorar el plano actitudinal -valorar del trabajo en equipo y liderazgo se les preguntó ¿quién asume generalmente el rol de liderazgo en el trabajo en equipo? el $68.00 \%$ opinó que es $\mathrm{H}-\mathrm{M}$; aunque el restante porcentaje muestra sesgo. Los $\mathrm{H}$ opinan que un $25.18 \%$ los $\mathrm{H}$ y $6.79 \%$ las $\mathrm{M}$; y las $\mathrm{M}$ opinan que el liderazgo lo asume una $\mathrm{M}$ en $24.10 \%$ y los $\mathrm{H}$ en $7.93 \%$. ¿Quién es mejor para liderar los trabajos por equipo?; el $70.52 \%$ opinan que tanto $\mathrm{H}-\mathrm{M} ; 19.82 \%$ las $\mathrm{M}$ y el restante $9.62 \%$ los $\mathrm{H}$. Por otro lado, los encuestados perciben que la responsabilidad mostrada en la entrega del trabajo en tiempo y forma se inclina más hacia las mujeres $(37 \% \mathrm{M}$ y el $44.29 \% \mathrm{H})$, al igual que la creatividad $(29.55 \% \mathrm{M}$ y $35.07 \% \mathrm{H})$.

\section{Plan de vida y carrera}

Para la exploración de los planes de vida y carrera de los encuestados se les presentaron seis metas, tres en el plano profesional y tres relacionadas con metas personales o familiares. Se les pidió indicar el orden de preferencia con respecto a dichas metas que tienen a cinco años de egresar de su respectiva carrera, los resultados fueron: Tener una maestría 29.95\%; Poner una empresa $27.96 \%$; Tener un puesto gerencial $22.60 \%$; Comprar una casa $17.03 \%$; Contraer matrimonio $1.52 \%$; y Tener hijos $0.95 \%$. Para todas las agrupaciones de los datos de la muestra se obtuvo que contraer matrimonio y tener hijos son las actividades con menor prioridad. Los datos agrupados por áreas de conocimiento se muestran en la tabla 5.

Tabla 5: Plan de vida y carrera por áreas de conocimiento en el IPN

\begin{tabular}{|l|l|l|l|}
\hline Prioridad & \multicolumn{1}{|c|}{ ICFM } & \multicolumn{1}{c|}{ CMB } & \multicolumn{1}{c|}{ CSA } \\
\hline \multirow{4}{*}{ Mayor } & Poner una empresa & Tener una maestría & Poner una empresa \\
\cline { 2 - 4 } & Tener una maestría & Comprar una casa & Tener una maestría \\
\cline { 2 - 4 } & Tener un puesto gerencial & Poner una empresa & Tener un puesto gerencial \\
\cline { 2 - 4 } Menor & Comprar una casa & Tener un puesto gerencial & Comprar una casa \\
\hline
\end{tabular}

Se les preguntó ¿en caso de formar una familia qué tan importante es para ti que ambos trabajen? 76.74\% de las $\mathrm{M}$ y $52.66 \%$ de los $\mathrm{H}$, respecto al total de $\mathrm{M} \mathrm{y} \mathrm{H}$, lo consideraron como muy importante; ambos pueden trabajar, pero no es tan importante con $20.30 \%$ para las $\mathrm{M}$ y $39.71 \%$ para los $\mathrm{H}$. La respuesta con menor frecuencia es que uno trabaje y el otro se dedique al hogar con $2.96 \%$ para $\mathrm{M}$ y $7.63 \%$ para $\mathrm{H}$. Se valoró la posibilidad de una relación entre estas variables con una prueba de $\mathrm{V}$-Cramer que nos arroja un valor de $\mathrm{V}_{c}$ $=0.253 \mathrm{lo}$ que nos indica que estos datos muestran un efecto pequeño. Al realizar el análisis para las diferentes áreas de conocimiento del IPN se encontró que hay diferencias entre ICFM y las otras dos -CMB, CSA- que entre ellas tienen resultados similares. Esta diferencia corresponde con la que señalamos entre mujer y hombre por lo que se afirma que está relacionada con la proporción de $\mathrm{M}$ y $\mathrm{H}$ inscritos en esas áreas.

Dentro de la misma categoría, se analizó la percepción que tienen los estudiantes sobre ¿quién gana más, H o M? ¿Tiene mejores puestos? y la preferencia por que su primer jefe sea $\mathrm{H}$ o M; el $97.11 \%, 69.10 \%, 79.72 \%$ responden que es indistinto y los porcentajes restantes siempre afirman que el hombre gana más y con un mayor porcentaje que tiene mejores puestos. Al analizar por área de conocimiento se detectó que los $\mathrm{H}$ de ICFM están más convencidos del hecho que los hombres tienen mejores puestos que las mujeres. También resulta que independientemente del área y del sexo los encuestados señalan una preferencia porque su jefe que sea del sexo contrario $17.53 \%$ contra los que desean que sea del mismo sexo $13.38 \%$. Respecto a la percepción de justicia en el ambiente laboral un $70.20 \%$ afirma que es injusto porque favorece a los $\mathrm{H}, 23.86 \%$ afirman que es justo y sólo el $5.94 \%$ asegura que es injusto porque favorece a las $\mathrm{M}$. Este comportamiento cambia cuando se analizan los datos por $\mathrm{H}$ o M, se reduce al $62.07 \%$ para los $\mathrm{H}$ que afirman que es injusto porque se favorece a los $\mathrm{H}$; o aumenta al $78.44 \%$ de las $\mathrm{M}$ que afirman lo mismo. Finalmente, se les preguntó ¿cuál será el sueldo que esperas ganar en tu primer empleo? Los $\mathrm{H}$ esperan un salario mayor, un promedio de $11,076.23 \pm 3,771.38$ y las M 9,443.25 $\pm 3,018$.33. No se encontró una diferencia significativa al analizar por áreas de estudio; es posible que haya una relación con el promedio académico, pero será necesario tomar datos más precisos para poder establecer una relación.

Para ponderar las respuestas a la pregunta de qué decepcionaría más a tus padres, se hizo un promedio de producto pesado $(0=$ no se decepcionan $1=$ se decepcionan) y se obtuvo que los encuestados manifiestan que sus padres se decepcionan poco 0.45 si no se casan y 0.49 si no llegan a tener hijos. En cambio, la decepción será 0.80 si no tiene éxito profesional y de 0.73 de no tener éxito económico. No se encontró diferencias por género ni por área de estudio.

Conciencia y apertura al cambio

Con la finalidad de aportar evidencia sobre la conciencia que está experimentando la sociedad relacionados con la igualdad entre $\mathrm{H}$ y $\mathrm{M}$ y el necesario cambio en los roles y estereotipos para que esto suceda, se cuestionó a los alumnos respecto a quién realiza actividades ligadas al estereotipo de ama de casa 
(actividades hogar: $\mathrm{AH}$ ): hacer la comida, realizar compras de alimentos, lavar ropa, limpiar el hogar y también sobre actividades de toma de decisiones (actividades decisión: AD): Decidir sobre actividades de fin de semana, tipo de auto, administrar dinero, planear vacaciones. Los resultados indican que: las AH son realizadas en un $64.03 \%$ por la madre y $14.48 \%$ por el encuestado y sólo $7.12 \%$ por el padre y 14.48 por hermanos $u$ otro. $Y$ las actividades AD son realizadas principalmente por el padre con $37.28 \%$ y la madre con $42.14 \%$. Es decir, que en la muestra analizada la mayor parte de las actividades (tanto las de hogar como las de decisión) se realizan por parte de la madre. Se analizó si los hombres encuestados viven una situación diferente a las de las mujeres realizando un análisis de chi cuadradas de Pearson y la transformamos en $\mathrm{V}$ de Cramer; se obtuvo un valor de $\mathrm{Vc}=0.192$, lo que indica una relación baja o inexistente; al igual que para las diferentes áreas del IPN y por $\mathrm{H}$ y M por cada área y por edad. Al analizar las respuestas de los encuestados respecto al sostén económico de su casa se obtuvo la información mostrada en la tabla 6.

Tabla 6: Porcentajes de actividades realizadas por integrantes de la familia respecto la persona que es el sostén económico principal de la casa

\begin{tabular}{|r|c|c|c|c|c|c|c|c|}
\hline Sostén & \multicolumn{4}{|c|}{ AH (actividades de hogar) } & \multicolumn{3}{c|}{ AD (actividades de decisión) } \\
\cline { 2 - 10 } económico & Madre & Padre & Encuestado & Otros & Madre & Padre & Encuestado & Otro \\
\hline Madre & $57.00 \%$ & $5.61 \%$ & $19.87 \%$ & $17.52 \%$ & $63.83 \%$ & $21.91 \%$ & $10.91 \%$ & $3.35 \%$ \\
\hline Padre & $69.57 \%$ & $8.18 \%$ & $10.89 \%$ & $11.36 \%$ & $33.56 \%$ & $50.50 \%$ & $5.21 \%$ & $10.73 \%$ \\
\hline
\end{tabular}

En estos datos se verificó el mismo comportamiento: las actividades que realiza la madre son superiores a las que realiza cualquier otro miembro de la familia y sólo cuando el padre es el sostén económico se involucra en la mitad de las actividades de decisión del hogar. En este caso al realizar la relación con la $V$ de Cramer obtenemos un valor de $V_{c}=0.513$ lo que permite concluir que existe una relación entre el sostén económico y las actividades desarrolladas. De igual forma se agruparon estos datos de acuerdo con el área de estudio del alumno, edad, sexo sin detectar otra relación. Por otra parte, cuando se preguntó a los alumnos sobre si consideraban que la división de actividades entre su padre y su madre es justa, el $61.74 \%$ responden que es una relación justa, el $18.97 \%$ que es injusta porque favorece al hombre; el $1.63 \%$ que es injusta porque favorece a la mujer y $17.66 \%$ señalaron la opción otro.

Finalmente, se preguntó a los encuestados sobre su opinión respecto a siete actividades y si éstas deberían ser realizadas por el padre, la madre, el hermano/a, el encuestado u otro. De las actividades cuatro se consideraron estereotipo femenino: $\mathrm{F} 1=$ Tareas domésticas, F2=cuidar a los hijos, F3=cuidar a los hermanos menores, F4=cuidar a los padres cuando sean mayores; y tres actividades de estereotipo masculino: M1=Reparaciones en el hogar, M2=Sostener económicamente el hogar y M3 = la seguridad del hogar. Se obtuvo que se asignan a la madre las actividades $\mathrm{F} 1$ con $43.30 \%$, F2 con $60.54 \%$ y $\mathrm{F} 3$ con $29.9 \%$; al padre se asignan M1 con $53.33 \%$, M2 con $63.16 \%$ y M3 con $58.28 \%$. La actividad F4 fue asignada con $47.19 \%$ al propio encuestado. El rol con menos actividades asignadas es el del hermano/a con 1.03\% \pm 1.02 .

La respuesta "otro" se consideró como que el encuestado no asignó la actividad ni a H ni a M y un $11.09 \%$ respondieron "otro" a las siete tareas $5.99 \%$ hombres y $5.10 \%$ mujeres. Lo que hizo suponer que se tiene evidencia de que las mujeres asocian más actividades al rol estereotipo que los hombres; para indagar sobre este posible resultado se cambió la respuesta madre por mujer, padre por hombre, encuestado por el sexo del encuestado y se ignoró las respuestas hermano/a (las menos asignadas). Así se contó para cada uno de los encuestados el número de actividades asignadas de acuerdo con el estereotipo, actividades $\mathrm{F}$ a mujer y actividades $\mathrm{M}$ a hombre; las que asigna contra el estereotipo, actividades $\mathrm{F}$ a hombre y actividades $\mathrm{M}$ a mujer y los que no asignan rol a las actividades. Los resultados se muestran en la tabla 7.

Tabla 7: Porcentajes de actividades realizadas por integrantes de la familia respecto la persona que es el sostén económico principal de la casa

\begin{tabular}{|r|c|c|c|c|c|c|c|c|}
\hline \multirow{2}{*}{$\begin{array}{c}\text { Actividad } \\
\text { (H=hombre, M= Mujer) }\end{array}$} & \multicolumn{7}{|c|}{ Cantidad de respuestas } \\
\cline { 2 - 9 } & 0 & 1 & 2 & 3 & 4 & 5 & 6 & 7 \\
\hline Coincide estereotipo(H) & $14.11 \%$ & $7.42 \%$ & $9.82 \%$ & $16.09 \%$ & $17.35 \%$ & $21.42 \%$ & $11.60 \%$ & $2.19 \%$ \\
\hline Coincide estereotipo(M) & $11.42 \%$ & $5.50 \%$ & $7.72 \%$ & $10.78 \%$ & $12.90 \%$ & $16.07 \%$ & $20.93 \%$ & $14.69 \%$ \\
\hline Contrario estereotipo(H) & $39.71 \%$ & $28.74 \%$ & $17.45 \%$ & $9.09 \%$ & $4.60 \%$ & $0.42 \%$ & $0.00 \%$ & $0.00 \%$ \\
\hline Contrario estereotipo(M) & $71.25 \%$ & $16.70 \%$ & $8.03 \%$ & $3.17 \%$ & $0.63 \%$ & $0.11 \%$ & $0.11 \%$ & $0.00 \%$ \\
\hline Otro(H) & $49.01 \%$ & $13.48 \%$ & $8.99 \%$ & $5.33 \%$ & $4.49 \%$ & $4.08 \%$ & $2.72 \%$ & $11.91 \%$ \\
\hline Otro(M) & $46.62 \%$ & $13.42 \%$ & $9.83 \%$ & $6.03 \%$ & $5.29 \%$ & $4.76 \%$ & $3.81 \%$ & $10.25 \%$ \\
\hline
\end{tabular}


Para cuantificar este resultado se hizo un promedio de producto pesado entre el número de respuestas y las frecuencias relativas de la respuesta, así cero implica no hay coincidencia con el estereotipo y 7 coincidencia total. Se tiene que los hombres coinciden con el estereotipo con un 3.37 mientras que las mujeres con un 4.14. Para el caso de estar en contra del estereotipo, los hombres tienen un valor de $1.11 \mathrm{y}$ las mujeres de 0.46 y respuestas "otro" tenemos un valor de 1.85 para hombres y 1.91 para mujeres. Sobre esta misma pregunta, pero agrupando por área de conocimiento, Figura 1, se tiene un comportamiento diferente por área.

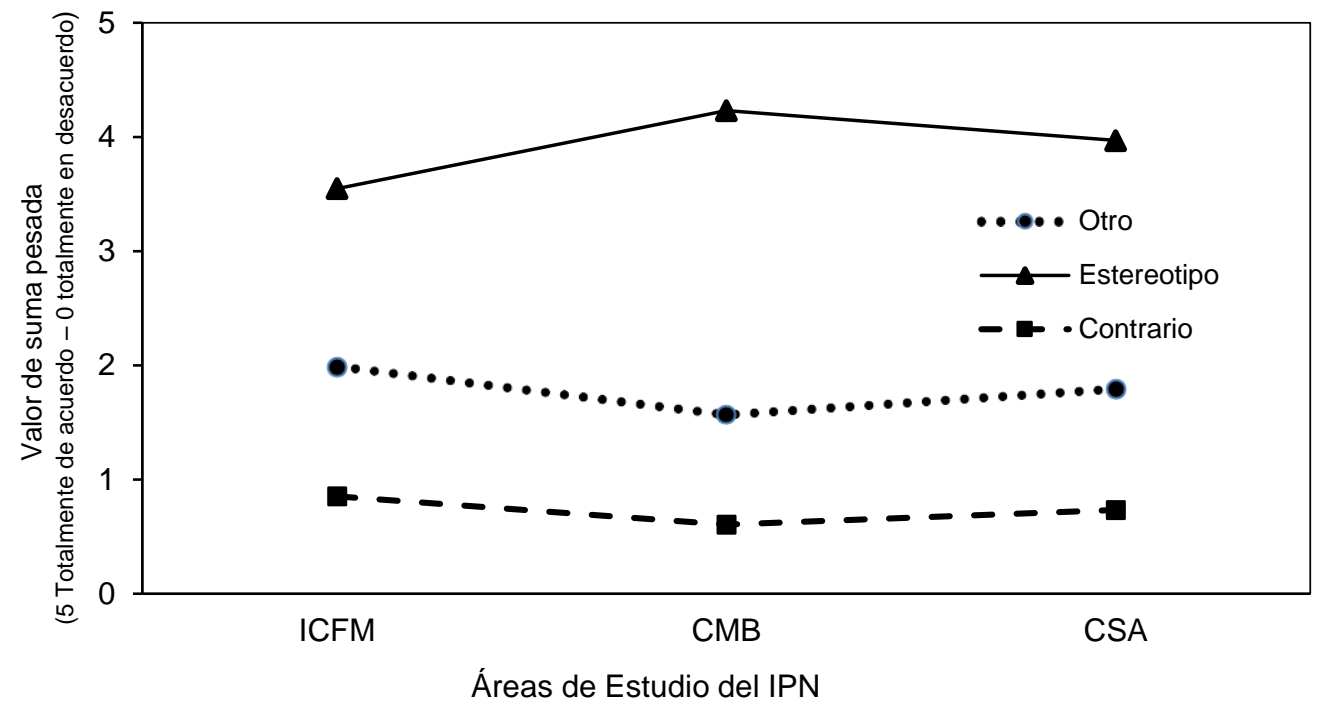

Fig. 1: Comportamiento de la muestra por área de conocimiento

Se puede ver que los $\mathrm{H}$ y M de ICFM tienden a no asignar un sexo a las actividades, que los alumnos de CSA reducen esta asignación, pero los de CMB son los que asignan más las actividades con un sexo y más frecuentemente al estereotipo. Se podría suponer que este comportamiento está influenciado porque en ICFM el número de mujeres es menor y por ello el comportamiento de baja coincidencia con el estereotipo y la más alta con el contrario, pero esto no explicaría por qué aumenta el valor de Otro para esta área de conocimiento. Además, el número de mujeres es mayor en CSA y en este caso se encuentra que quienes tienen más coincidencia con el estereotipo y menos en contra de él es CBM.

\section{DISCUSIÓN}

Este apartado se centra en la formación y el desarrollo de las competencias interpersonales y sistémicas del estudiantado de educación superior del IPN, concretamente las relacionadas con el trabajo en equipo y liderazgo; plan de vida y carrera y conciencia y apertura al cambio establecidas en el perfil de egreso (IPN, 2004); es importante enfatizar que el desempeño efectivo de los estudiantes activa de manera integrada conocimientos, habilidades, actitudes y valores (Beneitone et al. 2007; Villa y Poblete, 2011; Tejeda, 2016) pero por la naturaleza de la investigación en la discusión se abordarán de manera separada.

¿El desarrollo de las competencias interpersonales: Trabajo en equipo y actitud de liderazgo se está logrado en el IPN? Como uno de los bastiones del egresado del IPN (2004) se marca el desempeño efectivo en trabajos de equipo con actitudes de liderazgo; los resultados muestran marcadas diferencias por áreas de conocimiento; en general los estudiantes manifiestan que trabajan bien en equipo, no obstante la actitud de liderazgo está más desarrollada en los estudiantes de CSA. Esto significa que, principalmente, en las unidades académicas que imparten ICFM son las que requieren implementar pedagogías más flexibles que permitan fortalecer la formación integral del estudiante ((Tawil y Cougoureux, 2013; UNESCO, 2007); de lo contrario se estarían desatendiendo los componentes del perfil de egreso del IPN lo que podría perjudicar el pleno desarrollo profesional de los egresados. Las competencias interpersonales por definición más enfocadas en el saber estar de los estudiantes -actitudes- (Muñoz, Medina, y Guillén, 2016; Villarroel y Bruna, 2014; Farías y Firinguetti, 2012) deben desarrollarse en las IES a través de todo el mapa curricular (Villa y Poblete, 2011); no obstante se coincide con Macías et al. (2017) y Rojo y Navarro (2016) que la forma más adecuada para fomentar la focalización en el desarrollo de las competencias transversales es a través de la tutoría, porque los encuestados muestran diferentes percepciones relacionadas no solo con el liderazgo, sino con la responsabilidad en la entrega de trabajos en tiempo y forma o en la creatividad relacionada con ser $\mathrm{H}$ $\mathrm{M}$, la atención individualizada de los estudiantes promoverá mayor efectividad. 
¿El desarrollo de la competencia sistémica: plan de vida y carrera se está logrado en el IPN? Que los egresados tengan la habilidad para desarrollar su plan de vida y carrera (IPN, 2004) implica que los estudiantes de educación superior sean capaces de establecerse metas a futuro. De acuerdo con los resultados: tener un posgrado, poner una empresa o lograr un puesto gerencial son las que presentaron mayor preferencia por los estudiantes. De acuerdo con Farías y Firinguetti (2012) el espíritu emprendedor implica que los egresados generen acciones por iniciativa propia; por lo tanto en el IPN, aunque la matrícula muestra un comportamiento masculinizado y feminizado, sí se está desarrollando esta competencia (Barffusón et al., 2010; Zapata y Ayala, 2014) dado que el estudiantado muestra planes profesionales a futuro que parecieran estar más ligadas a una actuación autónoma (Bell, 2017) y al concepto de igualdad (ONU Mujeres, 2007). Sin embargo, existen factores ligados a la percepción de los estudiantes relacionados con los puestos de trabajo, tales como que los hombres tienes mejores puestos y perciben salarios más altos, o bien que existe injusticia en el mundo laboral porque se favorece a los hombres; lo que podrían desmotivar a las egresadas. Entonces se recomienda realizar más investigaciones cuyo objetivo sea fortalecer la capacidad de agencia de las estudiantes.

¿Qué actitudes persisten, sobre los roles y estereotipos, en los estudiantes de educación superior del IPN? Los roles y estereotipos fueron valorados en aspectos conductuales y afectivos (Le Pelley, et al. 2010 y ONU Mujeres, 2011); a nivel conductual se valoró la realidad de las actividades en las casas de los estudiantes y los resultados no muestran un cambio en ellos. Sin importar que las madres sean el sostén económico de la familia, seis de cada diez estudiantes manifestaron que sus madres realizan las actividades clasificadas como del hogar y de decisión; adicionalmente perciben como justa la división de tareas en su casa. Esto es preocupante, porque más allá de lo que sucede en su casa, o precisamente por ello, a nivel afectivo los estudiantes asignan la responsabilidad de realizar tareas en el hogar con el género; este comportamiento es menor para los estudiantes de ICFM, y los de CMB son los que mayormente manifiestan este comportamiento. Entonces los estudiantes no dan muestra de ser conscientes y abiertos al cambio (IPN, 2004; UNESCO, 2011); dicha conciencia está estrechamente relacionada con las competencias sistémicas por definición más enfocadas en el saber ser de los estudiantes -valores- (Muñoz, Medina, y Guillén, 2016; Villarroel y Bruna, 2014; Farías y Firinguetti, 2012). El colectivo docente del IPN debe incide desde la percepción que tienen los estudiantes de los roles y estereotipos como base para la transformación cultural y el respectivo cambio de actitudes, valores y prácticas, coadyuvando con la aspiración de una sociedad igualitaria (ONU Mujeres, 2011).

\section{CONCLUSIONES}

Con base en el análisis, los resultados de la investigación y la discusión se pueden obtener 5 conclusiones principales: 1) la competencia plan de vida y carrera sí se está desarrollando dado que los encuestados manifiestan planes profesionales a futuro ligados a una actuación autónoma; 2) la existencia de diferentes grados de desarrollo de la competencia trabajo en equipo y liderazgo -más desarrollada en estudiantes de CSA y menos en los de ICFM-; 3) existe un área de oportunidad con relación a la conciencia y apertura al cambio porque en la conducta de los estudiantes y en la valoración que hacen de diferentes actividades prevalecen roles y estereotipos -más arraigadas en estudiantes de CMB y menos en ICFM- aprendidos desde su socialización primaria y que el IPN no ha logrado transformar para reformular su vida cotidiana; 4) el programa de tutorías del IPN podría ser más efectivo para el desarrollo de competencias transversales en los estudiantes siguiendo dos principios: la prevención con base en un diagnóstico individualizado y la potenciación de habilidades, capacidades, actitudes y valores vinculados con estas competencias; 5) falta profundizar el conocimiento de la formación de las competencias transversales en investigaciones que aborden la percepción de los docentes y las metodologías para lograr el trabajo interdisciplinario asociado.

\section{AGRADECIMIENTOS}

Se agradece al Instituto Politécnico Nacional - Secretaria de Investigación y Posgrado las facilidades otorgadas para la realización de esta investigación.

\section{REFERENCIAS}

Babie, E., Fundamentos de investigación social, Thompson Internacional, D.F., México (2006)

Barffusón, R., J. Revilla y C. Carrillo, Aportes feministas a la educación, ISSN: 0185-1594, Enseñanza e investigación en psicología, 15(2), 357-376 (2010)

Bell, R., Pilares de la educación para el siglo XXI e inclusión educativa: interrelaciones y horizontes compartidos, Publicaciones Didácticas, núm. 90 (2017)

Beneitone, P., C. Esquetini y otros tres autores, (Eds.), Reflexiones sobre la Educación Superior en América Latina, Informe Final del Proyecto Tuning América Latina 2004-2007 (2007) 
Berger, P. y T. Luckman, La construcción social de la realidad, Amorrortú, Buenos Aires, Argentina (2006)

Bolívar, A., Las competencias básicas para la vida más transversales, Buenas prácticas para su tratamiento en el centro educativo y en el aula, USAID Ministerio de Guatemala, Guatemala, Guatemala (2011)

Bordieu, P., Capital cultural, escuela y espacio social, Siglo XXI, Buenos Aires, Argentina (2008)

Buquet, A., Transverzalización de la perspectiva de género en la educación superior. Problemas conceptuales y prácticos, Perfiles educativos IISUE-UNAM, XXXIII, Número Especial, 211- 225 (2011)

Díaz, V., Técnicas de análisis de datos para investigadores sociales, Aplicaciones prácticas con SPSS para Windows, Ra-Ma, Madrid, España (1999)

Farías, F. y L. Firinguetti, Valoración de las competencias genéricas de los trabajadores sociales en el Gran Concepción, Chile, R. Katál, Florianópolis, 15(2), 182-192 (2012)

Gross, M., Psicología: La ciencia de la mente y la conducta, 5aㅡ. Ed., Manual Moderno, Ciudad de México, México (2012) INEGI, Hombres y mujeres en México, INEGI, D.F., México (2015)

IPN, Dirección de Evaluación, Secretaría de Gestión Estratégica. Estadística Básica. Disponible en: https://goo.gl/xBjdks (consultado junio 2018)

IPN, Materiales para la reforma académica. Tomo I “Un nuevo Modelo para el Instituto Politécnico Nacional”, IPN, Ciudad de México, México (2004)

Le Pelley, M., S. Reimers y otros cuatro autores, Stereotype formation: Biased by association, doi: 10.1037/a0018210, Journal of Experimental Psychology, General, 139 (1), 138-161 (2010)

Macías, E., M. Rodríguez, L. Aguilera y S. Gil, Adquisición de competencias transversales a través de la tutoría en la universidad, e-ISSN: 1988-236x, La Cuestión Universitaria, 9, 88-107 (2017)

Muñoz, F., A. Medina y M. Guillén, Jerarquización de las competencias genéricas basadas en las percepciones de docentes universitarios, doi: 10/1016/j.eq.2015.11.002, Educ. quím., 27(2) 2, 126-132 (2016)

ONU Mujeres, Principios para el empoderamiento de las mujeres, La igualdad es un buen negocio, ONU, New York, USA (2011)

Rojo, R. y N. Navarro, Competencias genéricas adquiridas, según estudiantes de la carrera de salud, Investigación en Educación Médica, 5(19), 172-181 (2016)

Sánchez, M., M. Suárez y otros cinco autores, Estereotipos de género y valores sobre el trabajo entre los estudiantes españoles, doi: 10-4438/1988592X-RE-2011-355-027, Revista de Educación, 355, 331-354 (2011)

Tamayo, M., El proceso de investigación científica, 5a Ed., Limusa-Noriega, D.F., México (2003)

Tawil, S. y M. Cougoureux, Una mirada actual a la educación encierra un tesoro. Evaluar la influencia del informe Delors de 1996, Investigación y prospectiva en la educación. Contribución temática, 04 (2013)

Tejeda, R., Las competencias transversales, su pertinencia en la integralidad de la formación de los profesionales, ISSN: 2224-2643, Didac@lia: Didáctica y Educación, VII (6), Edición especial, 199-222 (2016)

UNESCO, Educación de Calidad para todos: Un asunto de derechos Humanos, Autor, Santiago de Chile (2007)

UNESCO, La educación encierra un tesoro, Informe final de la Comisión Internacional sobre la Educación para el siglo XXI presidido por Jaques Delors, Santillana, D.F., México (1996)

Vega, U., M. Figueroa y F. Del Real, Reflexiones sobre la formación de competencias transversales y digitales en institución de educación superior de México, ISSN: 1659-0104, Ensayos Pedagógicos, XII (1), 17-38 (2017)

Villa, M. y M. Poblete, Evaluación de competencias genéricas. Principios, oportunidades y limitaciones, Bordón Revista de Pedagogía, 63(1), 147-170 (2011)

Villarroel, V. y D. Bruna, Reflexiones en torno a las competencias genéricas en educación superior: Un desafío pendiente, ISSNe: 0718-6924, Psicoperspectivas: Individuo y Sociedad, 13(1), 23-34 (2014)

Zapata, E. y M. Ayala, Políticas de equidad de género: educación para una escuela libre de violencia, ISSN: 1665-0441, Ra Ximhai Revista de Sociedad y Desarrollo Sustentable, 10(7), 1-21 (2014) 